\title{
Giant Ascending Colonic Diverticulum Presenting With Intussusception
}

\author{
Ho Jin Kim, Jin Ha Kim, Ok In Moon, Kyung Jong Kim \\ Department of Surgery, Chosun University School of Medicine, Gwangju, Korea
}

Diverticular disease of the colon is a common disease, and its incidence is increasing gradually. A giant colonic diverticulum (GCD) is a rare entity and is defined as a diverticulum greater than $4 \mathrm{~cm}$ in size. It mainly arises from the sigmoid colon, and possible etiology is a ball-valve mechanism permitting progressive enlargement. A plain abdominal X-ray can be helpful to make a diagnosis initially, and a barium enema and abdominal computed tomography may confirm the diagnosis. Surgical intervention is a definite treatment for a GCD. We report a case of an ascending GCD presenting with intussusception in a young adult.

Keywords: Giant colonic diverticulum; Ascending colon; Intussusception; Surgical intervention

\section{INTRODUCTION}

Diverticular disease is a common disorder occurring in elderly people, and its incidence is increasing. No more than $20 \%$ of the patients with colonic diverticulum have symptoms, and of those who have symptoms, $25 \%$ experience complications such as abscess, perforation, stricture or fistula. Adult intussusception represents $5 \%$ of all cases of intussusception and accounts for only $1-5 \%$ of intestinal obstructions in adults [1]. Almost $90 \%$ of intussusceptions in adult are secondary to a carcinoma, polyp, stricture or benign neoplasm, including a colonic diverticulum [2]. A giant colonic diverticulum (GCD) is a rare entity of disease and is defined as a diverticulum greater than $4 \mathrm{~cm}$ in size [3]. More than $90 \%$ are antimesenteric and arise from the sigmoid colon [4]. Several GCDs have been reported, almost all of which developed at old age and in the sigmoid colon $[5,6]$. We report a case of a GCD, which was removed by using a laparoscopic colectomy. To our

Received: May 24, 2013・Accepted: July 30, 2013

Correspondence to: Kyung Jong Kim, M.D.

Department of Surgery, Chosun University Medical School,

309 Pilmun-daero, Dong-gu, Gwangju 501-759, Korea

Tel: +82-62-220-3983, Fax: +82-62-228-3441

E-mail: kjkim@chosun.ac.kr

(c) 2013 The Korean Society of Coloproctology

This is an open-access article distributed under the terms of the Creative Commons Attribution NonCommercial License (http://creativecommons.org/licenses/by-nc/3.0) which permits unrestricted noncommercial use, distribution, and reproduction in any medium, provided the original work is properly cited. knowledge, this is the first case of an ascending GCD in a young adult that suggests a congenital origin in pathogenesis and presented with intussusceptions.

\section{CASE REPORT}

A 30-year-old female was transferred to Chosun University Hospital with a 1-month history of left lower quadrant pain. The laboratory examination at admission was unremarkable. Physical examination revealed left lower quadrant tenderness with a palpable abdominal mass. Plain abdomen X-ray presented a gas-filled cavity in the middle right abdomen. The computed tomography scan from an outside hospital showed a target sign suggesting intussusception in the mid to lower colon on the left side (Fig. 1). The intussusception was soon resolved spontaneously without any procedure. To evaluate the lesion, we performed a colonoscopic examination, which revealed a huge submucosal tumor (SMT)-like lesion in the ascending colon (Fig. 2). The abdominal computed tomographic scan was checked again for further assessment of the SMT-like lesion and showed $4.2-\mathrm{cm}$-sized encapsulated fluid of high density with a thick wall on the right side of abdomen (Fig. 3). A laparoscopic right hemicolectomy was performed because of uncertainty of the diagnosis and for a one-stage treatment. On the operation findings, the lesion which was $5 \mathrm{~cm} \times 5 \mathrm{~cm}$ in size was located in the mid portion of the ascending colon and extended to the hepatic flexure. Also, multiple enlarged lymph nodes were found around the ileocolic and the middle colic vessel and were re- 


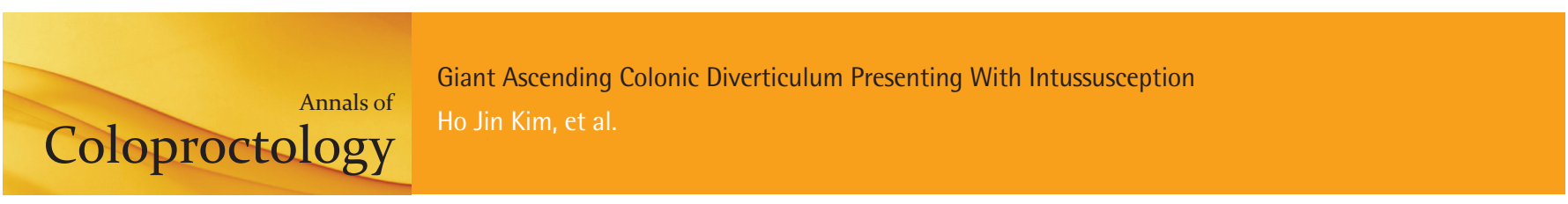

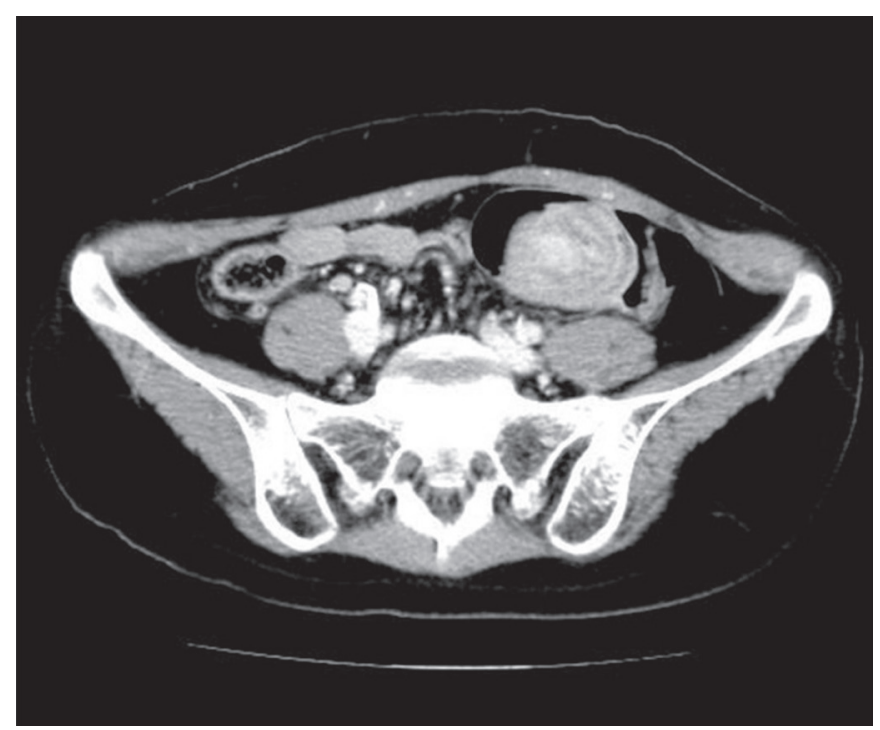

Fig. 1. Initial abdominal computed tomographic scan from an outside hospital showing the target sign in the left mid to lower abdomen.

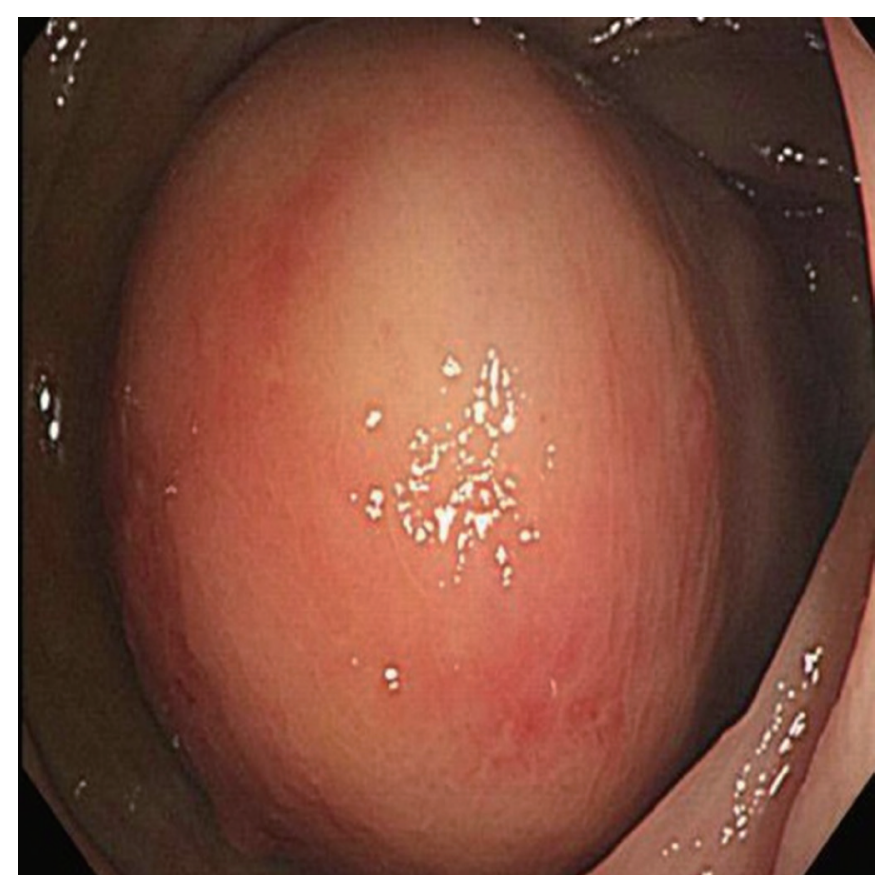

Fig. 2. Colonoscopic finding showing a large, smooth-surfaced, round mass lesion, mimicking a submucosal tumor, near the hepatic flexure.

moved by using an en bloc resection. Gross finding of the removed specimens showed a large diverticulum on the ascending colon (Fig. 4A). Histologic examination of the diverticulum showed the full thickness of the bowel wall from the mucosa to the serosa (Fig. $4 \mathrm{~B})$. The patient made a quick recovery and was discharged 7 days after surgery without any complication.

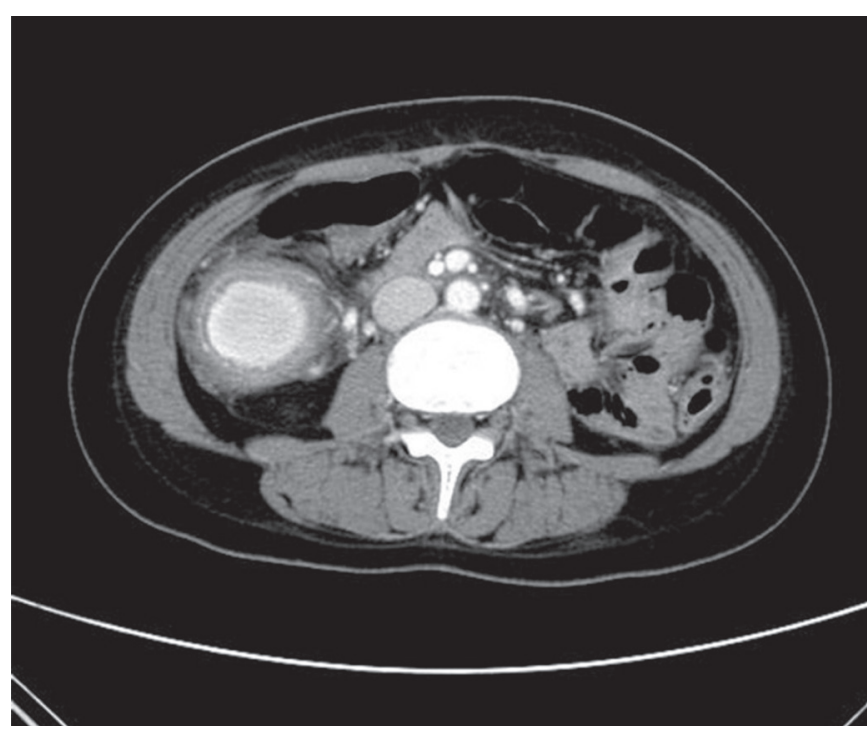

Fig. 3. Follow-up abdomen computed tomographic (CT) scan. Comparing to the previous CT scan, the large high-density mass had moved to the right side of the abdomen, suggesting an ascending colonic mass.

\section{DISCUSSION}

Colonic diverticular disease is a common condition and rarely presents as a singular giant diverticulum. A GCD, which is greater than $4 \mathrm{~cm}$ in size, is an uncommon manifestation of colonic diverticular disease and mainly occurs solitarily in the sigmoid colon $[5,7]$. The pathogenesis of a GCD is not well established. Possible theory supports a ball-valve mechanism through a tiny communicating neck, which causes air entrapment so that the diverticulum increases in size. Another theory postulates that the formation and the enlargement of a GCD is secondary to gas-forming organisms [8]. There are different types of GCDs. Steenvoorde et al. [9] described a histological classification of three distinct subtypes of GCDs. Type I is a pseudo-diverticulum containing remnants of the muscularis mucosa and the muscularis propria, and this type of diverticulum is found in colonic diverticular disease. Type II is an inflammatory GCD resulting from a local perforation of the mucosa with a persistent abscess cavity retaining contact with the colonic lumen, and the type III is a true diverticulum containing all layers of a normal bowel.

The clinical presentation of a GCD can be asymptomatic, but patients present most commonly with abdominal pain, constipation, or a palpable, nontender, abdominal mass $[9,10]$. A plain abdominal X-ray is used as the first line for diagnosis of a GCD. It is the simplest and most readily available tool and can reveal a large air-filled structure with or without fluid levels. A barium enema can demonstrate communication with the large bowel lumen in the majority of cases [11], but fails in approximately one-third of reported cases $[7,9]$. The differential diagnosis for a GCD in- 

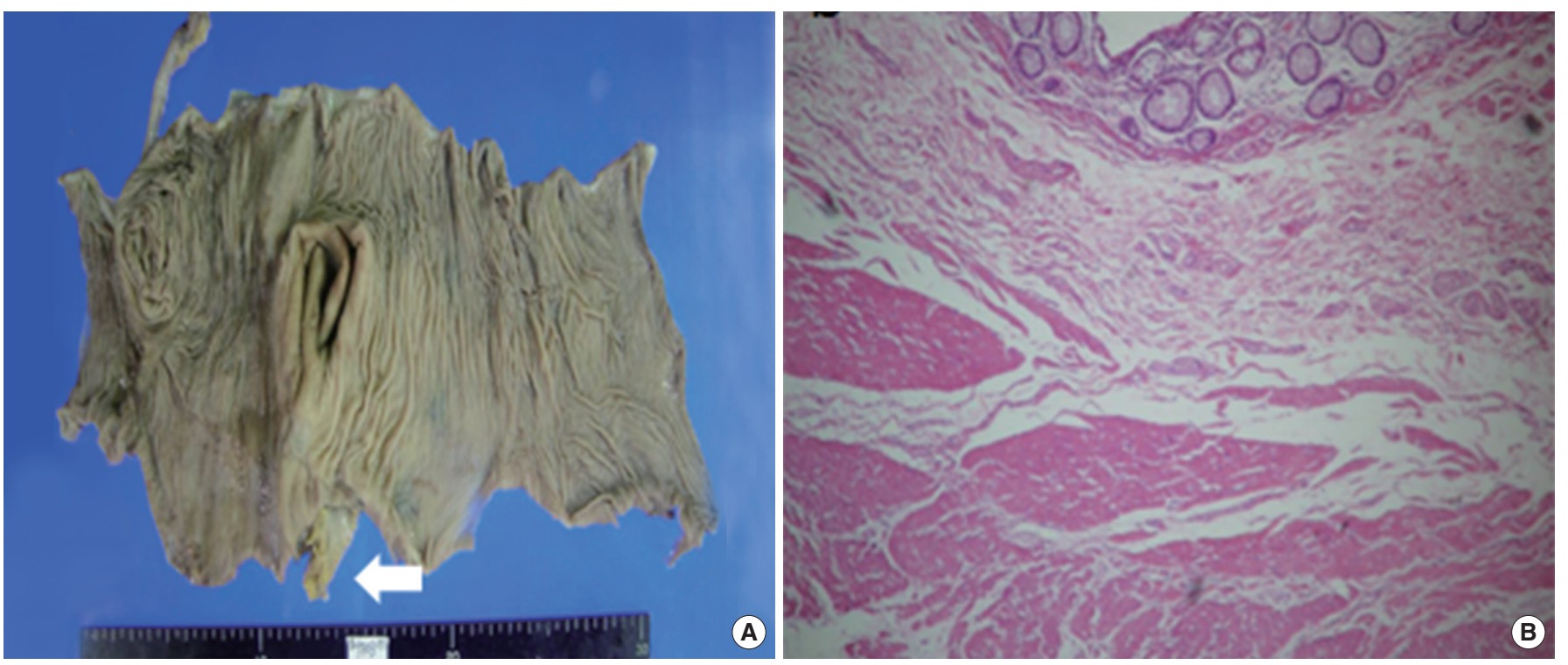

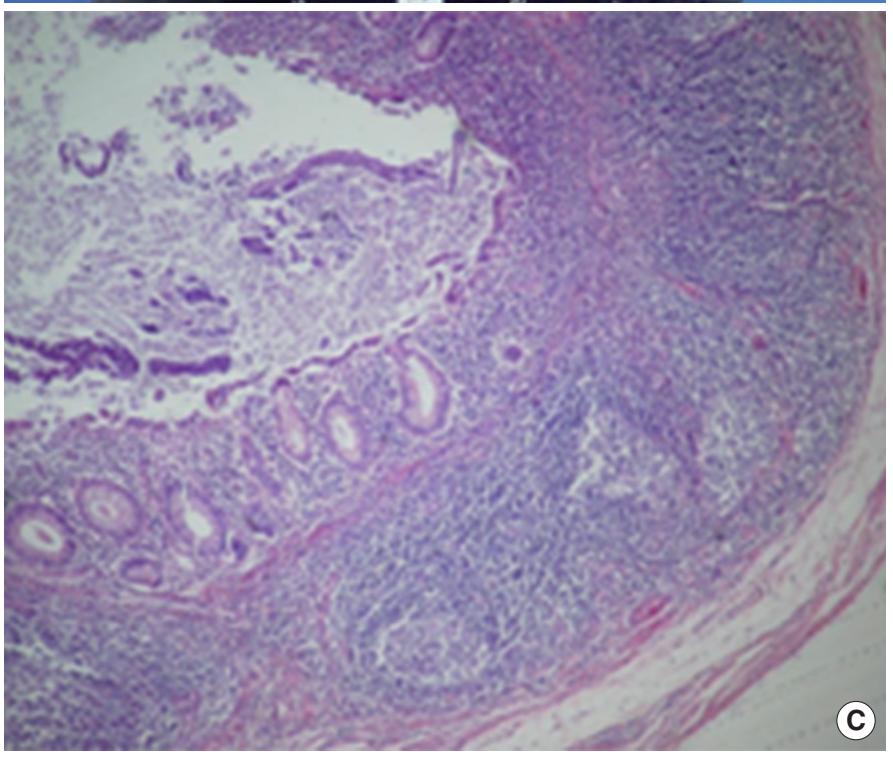

cludes sigmoid or caecal volvulus, a duplication cyst or giant Meckel diverticulum, and pneumatosis cystoides intestinalis [3].

A plain supine abdominal X-ray is the simplest and most readily available diagnostic tool and should be used as the first line in suspected cases because it can reveal a large air-filled structure (balloon-like appearance) with or without fluid levels. Computed tomography shows a smooth, thick-walled, air-filled structure intimately associated with the colon [8]. Colonoscopic evaluation is difficult in diagnosing a GCD. The ostium between the diverticulum and the colon is frequently too small to be detected [7-9]. GCD cases that have spontaneously resolved with conservative treatment have been reported; nevertheless, the gold standard of management of a GCD is a surgical resection and a primary anastomosis to exclude an underlying malignancy because adenocarcinomas arising from GCDs have been reported previously [9].
Fig. 4. Pathophysiology. Panel A shows a large diverticulum in the gross specimen. Panel B exhibits the true diverticulum and shows the full thickness of the bowel wall from the mucosa to the serosa in a low-power field $(\mathrm{H} \& \mathrm{E}, \times 100)$. The white arrow indicates an outpouching of the diverticulum. Panel $\mathrm{C}$ shows amorphous materials, consistent with a fecalith, in the diverticular lumen $(\mathrm{H} \& \mathrm{E}, \times 100)$.

In the era of minimal invasive surgery, a laparoscopic colonic resection including the lesion and a primary anastomosis may be feasible in most patients. Laparoscopic surgery has advantages of fast recovery, reduced hospital stay, less pain and better cosmesis $[11,12]$. Our case has some differences compared with previous cases. The lesion in our patient occurred in the ascending colon, not the sigmoid colon even though. The histological classification of our case was a true diverticulum, and the origin of diverticulum in our case might have been congenital because the patient was a young adult, unlike most GCD cases where the patients are much older. Our patient was treated with a laparoscopic right hemicolectomy, and there have been no symptoms or signs of recurrence since then (2 years). 


\section{CONFLICT OF INTEREST}

No potential conflict of interest relevant to this article was reported.

\section{REFERENCES}

1. Marinis A, Yiallourou A, Samanides L, Dafnios N, Anastasopoulos G, Vassiliou I, et al. Intussusception of the bowel in adults: a review. World J Gastroenterol 2009;15:407-11.

2. Weilbaecher D, Bolin JA, Hearn D, Ogden W 2nd. Intussusception in adults: review of 160 cases. Am J Surg 1971;121:531-5.

3. Beddy D, DeBlacam C, Mehigan B. An unusual cause of an acute abdomen: a giant colonic diverticulum. J Gastrointest Surg 2010; 14:2016-7.

4. Custer TJ, Blevins DV, Vara TM. Giant colonic diverticulum: a rare manifestation of a common disease. J Gastrointest Surg 1999; 3:543-8.

5. Kang JY, Hoare J, Tinto A, Subramanian S, Ellis C, Majeed A, et al. Diverticular disease of the colon--on the rise: a study of hospi- tal admissions in England between 1989/1990 and 1999/2000. Aliment Pharmacol Ther 2003;17:1189-95.

6. Kang JY, Melville D, Maxwell JD. Epidemiology and management of diverticular disease of the colon. Drugs Aging 2004;21:211-28.

7. Levi DM, Levi JU, Rogers AI, Bergau DK, Wenger J. Giant colonic diverticulum: an unusual manifestation of a common disease. Am J Gastroenterol 1993;88:139-42.

8. de Oliveira NC, Welch JP. Giant diverticula of the colon: a clinical assessment. Am J Gastroenterol 1997;92:1092-6.

9. Steenvoorde P, Vogelaar FJ, Oskam J, Tollenaar RA. Giant colonic diverticula: review of diagnostic and therapeutic options. Dig Surg 2004;21:1-6.

10. Scott DA, Glancy S. Spontaneous resolution of a giant colonic diverticulum. Clin Radiol 2008;63:833-5.

11. Choong CK, Frizelle FA. Giant colonic diverticulum: report of four cases and review of the literature. Dis Colon Rectum 1998; 41:1178-85.

12. Galandiuk S, Fazio VW. Pneumatosis cystoides intestinalis: a review of the literature. Dis Colon Rectum 1986;29:358-63. 